\title{
A PRIORI BOUNDS FOR ELLIPTIC OPERATORS IN WEIGHTED SOBOLEV SPACES
}

\author{
Serena Boccia, Maria Salvato And Maria Transirico
}

Abstract. This paper is concerning with the study of a class of weight functions and their properties. As an application, we prove some a priori bounds for a class of uniformly elliptic second order linear differential operators in weighted Sobolev spaces.

Mathematics subject classification (2010): 35J25, 35B65, 35R05.

Keywords and phrases: Weight functions, weighted Sobolev spaces, elliptic operators, a priori bounds.

\section{REFERENCES}

[1] S. Boccia, L. CASo, Interpolation inequalities in weighted Sobolev spaces, J. Math. Inequal. 2 (2008), 309-322.

[2] L. Caso, P. Cavaliere, M. Transirico, Solvability of the Dirichlet problem in $W^{2, p}$ for elliptic equations with discontinuous coefficients in unbounded domains, Matematiche (Catania) 57 (2002), 287-302.

[3] L. Caso, P. Cavaliere, M. Transirico, Uniqueness results for elliptic equations with VMOcoefficients, Int. J. Pure Appl. Math. 13 (2004), 499-512.

[4] L. Caso, P. Cavaliere, M. Transirico, An existence result for elliptic equations with VMOcoefficients, J. Math. Anal. Appl. 325 (2007), 1095-1102.

[5] L. CASo, M. Transirico, A priori estimates for elliptic equations in weighted Sobolev spaces, Math. Inequal. Appl. 13, 3 (2010), 655-666.

[6] P. Cavaliere, M. Longobardi, A. Vitolo, Imbedding estimates and elliptic equations with discontinuous coefficients in unbounded domains, Le Matematiche 51 (1996), 87-104.

[7] F. Chiarenza, M. Frasca, P. LONGO, Interior $W^{2, p}$ estimates for non divergence elliptic equations with discontinuous coefficients, Ricerche Mat. 40 (1991), 149-168.

[8] F. ChiAREnZA, M. Frasca, P. LONGO, $W^{2, p}$-solvability of the Dirichlet problem for nondivergence elliptic equations with VMO coefficients, Trans. Amer. Math. Soc. 336 (1993), 841-853.

[9] A. V. Glushak, M. Transirico, M. Troisi, Teoremi di immersione ed equazioni ellittiche in aperti non limitati, Rend. Mat. (7) 9 (1989), 113-130.

[10] C. Miranda, Sulle equazioni ellittiche del secondo ordine a coefficienti discontinui, Ann. Mat. Pura Appl. (4) 63 (1963), 353-386.

[11] M. Transirico, M. Troisi, Equazioni ellittiche del secondo ordine di tipo non variazionale in aperti non limitati, Ann. Mat. Pura Appl. (4) 152 (1988), 209-226.

[12] M. Transirico, M. Troisi, Ulteriori contributi allo studio delle equazioni ellittiche del secondo ordine in aperti non limitati, Boll. Un. Mat. Ital. (7) 4-B (1990), 679-691.

[13] M. Transirico, M. Troisi, A. Vitolo, Spaces of Morrey type and elliptic equations in divergence form on unbounded domains, Boll. Un. Mat. Ital. (7) 9-B (1995), 153-174.

[14] M. Transirico, M. Troisi, A. Vitolo, BMO spaces on domains of $\mathbb{R}^{n}$, Ricerche Mat. 45 (1996), 355-378.

[15] M. Troisi, Su una classe di spazi di Sobolev con peso, Rend. Accad. Naz. Sci. XL Mem. Mat. 10 (1986), 177-189.

[16] C. VitAnZA, $W^{2, p}$-regularity for a class of elliptic second order equations with discontinuous coefficients, Matematiche (Catania) 47 (1992), 177-186. 
[17] C. VitAnZA, A new contribution to the $W^{2, p}$-regularity for a class of elliptic second order equations with discontinuous coefficients, Matematiche (Catania) 48 (1993), 287-296. 